\title{
Relationship between MPB and Emergence of Structural Boundary in Cubic Phase of Pb-Based Perovskite-Type Solid Solutions PZT and PZN-PT
}

\author{
Yoshihiro Terado ${ }^{a}$, Chikako Moriyoshi ${ }^{\mathrm{a}}$, Yoshihiro Kuroiwa ${ }^{\mathrm{a}, \mathrm{d},{ }^{*} \text {, }}$ \\ Yasuhisa Yamamura ${ }^{\mathrm{b}}$ and Makoto Iwata ${ }^{\mathrm{c}}$ \\ ${ }^{a}$ Department of Physical Science, Hiroshima University, Kagamiyama, Higashi-Hiroshima, Hiroshima 739-8526, Japan \\ ${ }^{b}$ Department of Chemistry, University of Tsukuba, Tennodai, Tsukuba, Ibaraki 305-8571, Japan \\ ${ }^{\circ}$ Department of Engineering Physics, Electronics and Mechanics, Nagoya Institute of Technology, \\ Showa-ku, Nagoya, Aichi 466-8555, Japan \\ ${ }^{d}$ CREST, Japan Science and Technology Agency, Honcho, Kawaguchi, Saitama 332-0012, Japan \\ "Fax: 81-82-424-7398, e-mail: kuroiwa@sci.hiroshima-u.ac.jp
}

High-energy synchrotron-radiation powder-diffraction experiments for $\mathrm{PbZr}_{1-x} \mathrm{Ti}_{x} \mathrm{O}_{3}$ (PZT: $0 \leq x \leq$ 1) and $(1-x) \mathrm{Pb}\left(\mathrm{Zn}_{1 / 3} \mathrm{Nb}_{2 / 3}\right) \mathrm{O}_{3}-x \mathrm{PbTiO}_{3}(\mathrm{PZN}-\mathrm{PT}: 0 \leq x \leq 0.3)$ have been performed to investigate the relationship between the structural features of the cubic phase at high temperatures and the morphotropic phase boundary (MPB) at low temperatures. Our precise Rietveld analyses for PZT and PZN-PT with various compositions have revealed that the $\mathrm{Pb}$ atom in the cubic phase is disordered around the cubic corner site, and the thermal motions abruptly change at the distinctive composition $x \sim 0.5$ for PZT and at $x \sim 0.15$ for PZN-PT, where MPB of each solid solution separates the tetragonal and rhombohedral phases at low temperatures. No anomalous change in thermal motions is detected for other atoms. Hence, we consider that the appearance of MPB is strongly related to the change in local environment around the $\mathrm{Pb}$ atom.

Key words: PZT, PZN-PT, synchrotron radiation, powder diffraction, morphotropic phase boundary (MPB), tolerance factor

\section{INTRODUCTION}

$\mathrm{Pb}$-based perovskite-type solid solutions, $\mathrm{Pb}\left(B^{\prime}{ }_{1-x} B{ }^{\prime \prime}\right) \mathrm{O}_{3}$, have given rise to our interest for a long time, because of their high electromechanical coupling constant and piezoelectric strain constant. The prototype structure of the paraelectric phase in a high-temperature region is a cubic structure for the whole composition range of $x$, whereas the ferroic phases in a low-temperature region are essentially classified into some phases with different crystal symmetries. These ferroic phases can be separated by a morphotropic phase boundary (MPB). The excellent electromechanical properties are outstanding near MPB compositions.

$\mathrm{PbZr}_{1-x} \mathrm{Ti}_{x} \mathrm{O}_{3} \quad(\mathrm{PZT})$ and $(1-x) \mathrm{Pb}\left(\mathrm{Zn}_{1 / 3} \mathrm{Nb}_{2 / 3}\right) \mathrm{O}_{3}$ $x \mathrm{PbTiO}_{3}(\mathrm{PZN}-\mathrm{PT})$ are both well-known as piezoelectric ceramics for the industrial applications. PZT displays a complicated temperature-composition phase-diagram including various kinds of ferroic phases [1]. In a low-temperature region, ferroelectric phases cover most of the composition region, while an antiferroelectric phase with an orthorhombic symmetry appears only in the region close to the end member $\mathrm{PbZrO}_{3}(x=0)$. The ferroelectric phases are basically composed of two phases, that is, a rhombohedral phase in the Zr-rich region $(0<x<0.5)$ and a tetragonal phase in the Ti-rich region $(0.5<x \leq 1)$. In the PZN-PT system, the ferroic phases are also divided into two phases similar to the PZT system $[2,3]$, that is, rhombohedral close to PZN side $(0 \leq x<0.08)$ and tetragonal in the other large side $(0.08<x \leq 1)$.

The crystal structure of PZT and PZN-PT in the paraelectric phase has generally been considered to be isomorphous to the classical cubic perovskite structure, where $\mathrm{Pb}$ atom is settled at the cubic corner and $\mathrm{Zr} / \mathrm{Ti}$ or $\mathrm{Zn} / \mathrm{Nb} / \mathrm{Ti}$ at body center, and $\mathrm{O}$ atoms at the face centers In our previous studies, however, we demonstrated that the $\mathrm{Pb}$ atom in $\mathrm{PbZrO}_{3}$ in the paraelectric phase is disordered at 12 equivalent sites in the $\langle 110\rangle$ directions from the cubic corner site, while the $\mathrm{Pb}$ atom in $\mathrm{PbTiO}_{3}$ is ordered at the corner [4]. The ab initio calculations suggest that changes in $\mathrm{Zr} / \mathrm{Ti}$ composition give rise to phase transition of PZT through changes in populations of various local $\mathrm{Pb}$ environments, unlike of $\mathrm{Zr} / \mathrm{Ti}$ environments [5].

Recently, we have accurately analyzed the cubic structures of PZT and PZN-PT using high-energy synchrotron-radiation (SR) powder-diffraction $[6,7]$. In this paper, we summarize these results on the crystal structure analyses, and discuss the relationship between the structural features of the cubic structures and the existence of MPB caused by the $B$-site substitution for the PZT and PZN-PT solid solutions.

\section{EXPERIMENTAL}

The solid solutions of PZT $(x=0,0.15,0.25,0.35$, $0.48,0.55,0.6,0.65,0.75$ and 1$)$, except $\mathrm{PbZrO}_{3}(x=0)$, were synthesized by a conventional solid-state reaction at high temperatures. The starting materials were $\mathrm{PbO}$, $\mathrm{TiO}_{2}$ and $\mathrm{ZrO}_{2}$ powders. The $\mathrm{PbZrO}_{3}$ powder was purchased from Johnson Matthey. Single crystals of PZN-PT ( $x=0,0.05,0.08,0.2$ and 0.3 ) were grown by the flux method from a $\mathrm{PbO}-\mathrm{ZnO}-\mathrm{Nb}_{2} \mathrm{O}-\mathrm{TiO}_{2}$ mixture. The technical details of the synthetic processes for PZT 


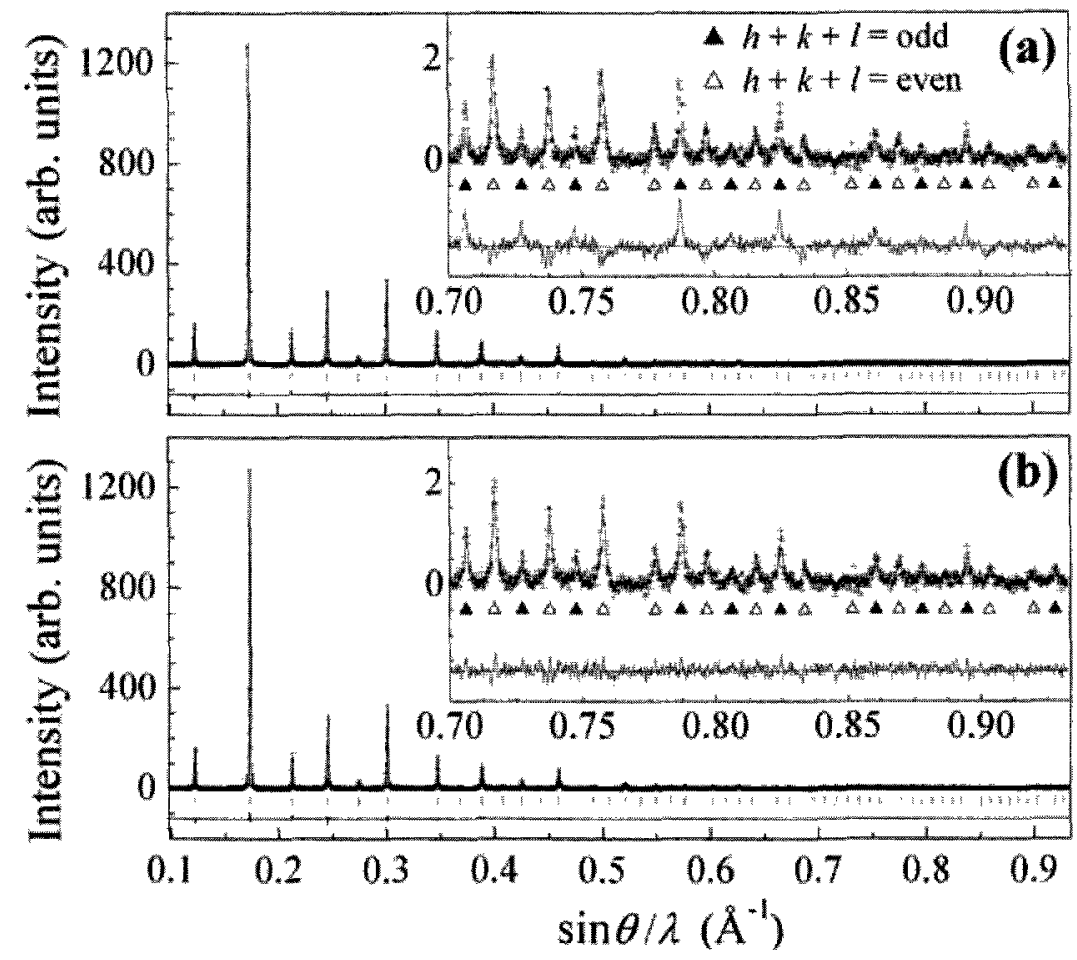

Fig. 1. Rietveld profile fitting result of $\mathrm{PZN}$ at $650 \mathrm{~K}$ in cubic phase based on different crystal structure models. (a) $\mathrm{Pb}$ atom ordered at $(0,0,0)$ position, (b) $\mathrm{Pb}$ atom disordered at twelve $(x, x, 0)$ equivalent positions. The systematic discrepancy between observed intensities $(+)$ and calculated intensities (solid line), obvious in the high-angle region of (a), is improved in (b).

and PZN-PT are described in the previous papers $[6,8]$. After grinding these PZT and PZN-PT samples in an agate mortar, the homogeneous granularity of the sample powder, which gave a homogeneous intensity distribution in the Debye-Scherrer powder ring, was achieved by the precipitation method. The sample powder was sealed in a quartz capillary of $0.2 \mathrm{~mm}$ internal diameter.

High-energy SR powder diffraction experiments were performed using a large Debye-Scherrer camera installed at BL02B2 in SPring-8 [9]. An imaging plate was used as a two-dimensional detector. High-energy diffraction experiments enable us to measure a large amount of diffraction data in a large reciprocal space, which was essential in this study for observing small displacements of atoms from their ideal positions, originating from atomic thermal vibration or disorder. Both extinction and absorption effects could be ignored in high-energy powder diffraction experiments, which was also very useful for the analysis of specimens such as PZT and PZN-PT that contain an extremely heavy element $\mathrm{Pb}$ and a light element $\mathrm{O}$. Furthermore, because of the high brilliance of SR, we could obtain the diffraction data with a good counting statistic in a short time. In the present study, diffraction intensity data with $d$-spacing of $0.55 \AA$ and above $\left(\sin \theta / \lambda<0.90 \AA^{-1}\right)$ were accumulated using high-energy $\mathrm{SR}$ with a wavelength of $0.356 \AA(\sim 35 \mathrm{keV})$, and analyzed by the maximum entropy method (MEM)/Rietveld method [10]. The sample temperature was controlled in the cubic phase by a $\mathrm{N}_{2}$ gas flow system at $850 \mathrm{~K} \pm 0.1 \mathrm{~K}$ for PZT and at $650 \mathrm{~K} \pm 0.1 \mathrm{~K}$ for PZN-PT.

\section{RESULTS AND DISCUSSION}

The crystal structures of PZT and PZN-PT in the cubic phase were analyzed by the Rietveld method. First, we assumed an ideal cubic perovskite structure, i.e., all constituent atoms are ordered at the special Wyckoff positions, $\mathrm{Pb}$ at $(0,0,0), \mathrm{Zr} / \mathrm{Ti}$ and $\mathrm{Zn} / \mathrm{Nb} / \mathrm{Ti}$ randomly at $(1 / 2,1 / 2,1 / 2)$, and $O$ at $(1 / 2,1 / 2,0)$ and equivalent positions in the space group of $P m \overline{3} m$ with harmonic thermal parameters. The profile fitting result of $P Z N$ is shown in Fig. 1(a) as a typical example. The obtained reliability factors $R_{\mathrm{WP}}, R_{\mathrm{I}}$ and $R_{F}$, based on the weighted profile, Bragg intensity and the structure factor, respectively, were not large, $R_{\mathrm{WP}}=2.52 \%, R_{\mathrm{I}}=2.04 \%$ and $R_{F}=4.85 \%$. However, $R_{F}$ was relatively larger than $R_{\mathrm{WP}}$ and $R_{\mathrm{I}}$. Furthermore, as depicted in the inset of Fig. 1 (a), a systematic discrepancy between the observed and calculated intensities was found. For $h+k+l=$ odd reflections ( $\boldsymbol{\Delta}$ marks), calculated intensities are consistently lower than observed intensities, whereas for $h+k+l=$ even reflections ( $\triangle$ marks), the situation is reversed. Such discrepancies were observed in other PZN-PT and PZT samples with different compositions. The discrepancies are particularly prominent in the high-angle region. This feature is highly similar to the cases of $\mathrm{PbZrO}_{3}$ [4] in the paraelectric phase which we previously reported. It is natural to expect that the heavy $\mathrm{Pb}$ atom is disordered around the $(0,0,0)$ position in the relevant directions. Accordingly, we assumed three types of structure models involving the disordered $\mathrm{Pb}$ 
atom in the $\langle 100\rangle,\langle 110\rangle$ and $\langle 111\rangle$ directions, and then reanalyzed the crystal structures using the Rietveld method adopting the split-atom method. In the $\langle 100\rangle$, $\langle 110\rangle$ and $\langle 111\rangle$ disorder models, one $\mathrm{Pb}$ atom is assumed to randomly occupy the $6(x, 0,0), 12(x, x, 0)$ and $8(x, x, x)$ equivalent positions, respectively, around the $(0,0,0)$ position. The reliability factors $R_{F}$ obtained from the profile fitting of PZN are significantly improved to $2.45 \%, 1.53 \%$ and $1.86 \%$ for the $\langle 100\rangle$, $\langle 110\rangle$ and $\langle 111\rangle$ disorder models, respectively. Among these three models, $R_{F}$ for the $\langle 110\rangle$ disorder model is the lowest, so that the twelve-site disorder model is preferable to the other two models for PZN. Figure $1(\mathrm{~b})$ shows the final profile fitting result of $P Z N$ in the cubic phase, for which the $\langle 110\rangle$ disorder model is assumed. We can see that the systematic discrepancy between observed and calculated intensities shown in Fig. 1(a) is reduced, as shown in the inset of Fig. 1(b). No disordered feature is detected for other atoms in the Rietveld analysis. The compositions calculated from the refined occupancies were in good agreement with the nominal compositions within a standard error of $1 \%$.

The crystal structure analyses were also carried out in a similar way for PZN-PT samples with other compositions. The lowest $R_{F}$ values were achieved by the $\langle 110\rangle$ disorder model for PZN-PT with $0 \leq x \leq 0.08$, whereas the $\langle 111\rangle$ disorder model was preferable for PZN-PT with $0.2 \leq x \leq 0.3$. Thus, we considered that the disordered directions of the $\mathrm{Pb}$ atom are changed at approximately $15 \% \mathrm{Ti}$ content in the PZN-rich region. A similar tendency was found in PZT system. The $\langle 111\rangle$ and $\langle 110\rangle$ disorder models gave the best fit for PZT with $0.15 \leq x \leq 0.48$ and $0.55 \leq x \leq 0.75$, respectively.
Accordingly, the disordered directions of the $\mathrm{Pb}$ atom are changed at approximately $50 \% \mathrm{Ti}$ content.

Subsequent to the Rietveld analysis, the charge density distributions were analyzed by the MEM/Rietveld method. The 71 independent structure factors obtained from the Rietveld analysis were used in the MEM analysis. The MEM analysis was carried out with the unit cell divided into $81 \times 81 \times 81$ pixels. The volume of one pixel corresponds to about $0.05 \times 0.05 \times$ $0.05 \AA^{3}$. The three-dimensional charge density distribution of cubic PZN at $650 \mathrm{~K}$ is shown in Fig. 2, where the depicted cell size is $2 a \times 2 b \times 2 c$. The one-dimensional electron density profiles of the $\mathrm{Pb}$ and $\mathrm{Zn} / \mathrm{Nb}$ atoms in the [110] direction are also shown. It appears to be a flat-top distribution in the charge density profile of the $\mathrm{Pb}$ atom compared with that of the $\mathrm{Zn} / \mathrm{Nb}$ atom, which supports the $\mathrm{Pb}$ disordered model evaluated from the Rietveld analysis. The electron density distributions around the $\mathrm{O}$ atoms are slightly extended in the directions perpendicular to the $\mathrm{Zn} / \mathrm{Nb}-\mathrm{O}$ covalent bond.

Figure 3 shows the structure parameters of $\mathrm{PZT}$ and PZN-PT at $850 \mathrm{~K}$ and $650 \mathrm{~K}$, respectively, which are obtained by the Rietveld analysis assuming the $\mathrm{Pb}$ 'ordered' model. The lattice parameter of the cubic structure is denoted by $a$, and $U_{\mathrm{Pb}}, U_{\mathrm{Zr} / \mathrm{Ti}}, U_{\mathrm{Zn} / \mathrm{Nb} / \mathrm{Ti}}$ and $U_{0}$ are the mean square thermal displacement parameters of $\mathrm{Pb}, \mathrm{Zr} / \mathrm{Ti}, \mathrm{Zn} / \mathrm{Nb} / \mathrm{Ti}$ and $\mathrm{O}$ atoms, respectively. The parameters $U_{\mathrm{Pb}}, U_{\mathrm{Zr} / \mathrm{Ti}}$ and $U_{\mathrm{Zn} / \mathrm{Nb} / \mathrm{Ti}}$ are crystallographically isotropic whereas $U_{0}$ can be converted to anisotropic parameters, $U_{\mathrm{O} 11}$ and $U_{\mathrm{O} 22}$, where $U_{011}$ is the parameter related to the thermal motion of an $\mathrm{O}$ atom along the $\mathrm{Zr} / \mathrm{Ti}-\mathrm{O}$ or $\mathrm{Zn} / \mathrm{Nb} / \mathrm{Ti}-\mathrm{O}$ bond and $U_{\mathrm{O} 22}$ is the parameter perpendicular to the

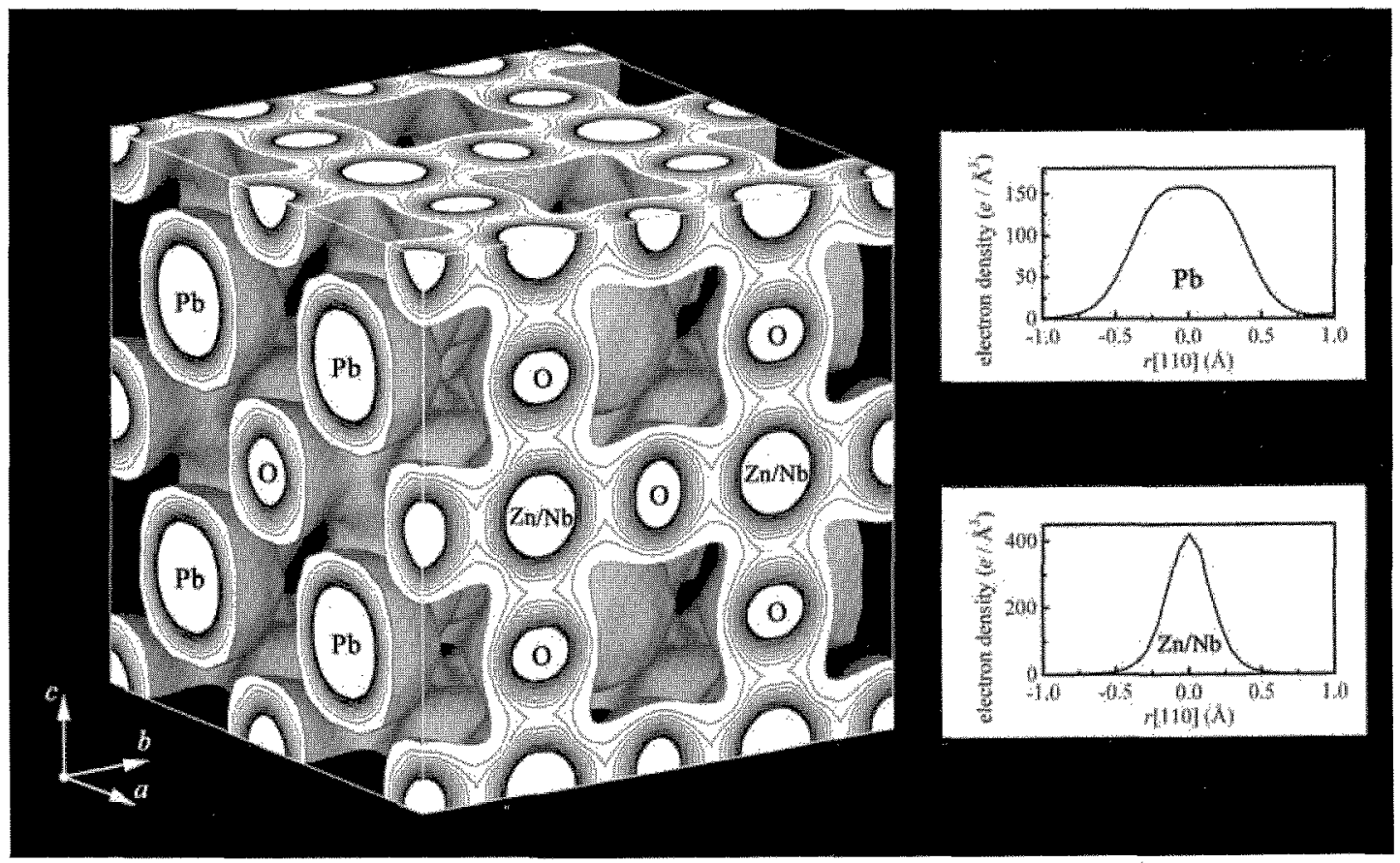

Fig. 2. MEM electron density distributions of $P Z N$ at $650 \mathrm{~K}$. The level of the equi-density surface of the three-dimensional map is $0.28 e / \AA^{3}$. The contour lines are drawn from $0.28 e / \AA^{3}$ to $4.08 e / \AA^{3}$ with $0.2 e / \AA^{3}$ intervals. Electron density profiles of $\mathrm{Pb}$ and $\mathrm{Zn} / \mathrm{Nb}$ along the [110] direction are depicted in the insets. 
bond. As shown in Fig. 3, the lattice parameter $a$ decreases linearly with increasing Ti content for both materials. The magnitude of $U_{\mathrm{OI}}$ is larger than that of $U_{\mathrm{O} 22}$. This anisotropic thermal motion of $\mathrm{O}$ atom is attributed to the deference of chemical bonding for each direction. Furthermore, note that the magnitude of $U_{\mathrm{Pb}}$ is fairly large as compared with those of $U_{\mathrm{Zt} / \mathrm{T}}, U_{\mathrm{Zn} / \mathrm{Nb} / \mathrm{Ti}}$ and $U_{\mathrm{O}}$, and $U_{\mathrm{Pb}}$ changes abruptly around $50 \% \mathrm{Ti}$ content for $\mathrm{PZT}$ and $15 \% \mathrm{Ti}$ content for PZN-PT, respectively. At these compositions, disordered directions of the $\mathrm{Pb}$ atom are also significantly changed. Thus, we consider that structural boundary can be defined even in the cubic phase for the change in disordered characteristics of the $\mathrm{Pb}$ atom. In Fig. 3, the structural boundary is depicted on the phase diagram by a dotted line. It is as if the boundary lies just above MPB.

As shown in Fig. 3, it seems that the structural boundary detected in the cubic phase is strongly related to MPB. Since the emergence of the structural boundary
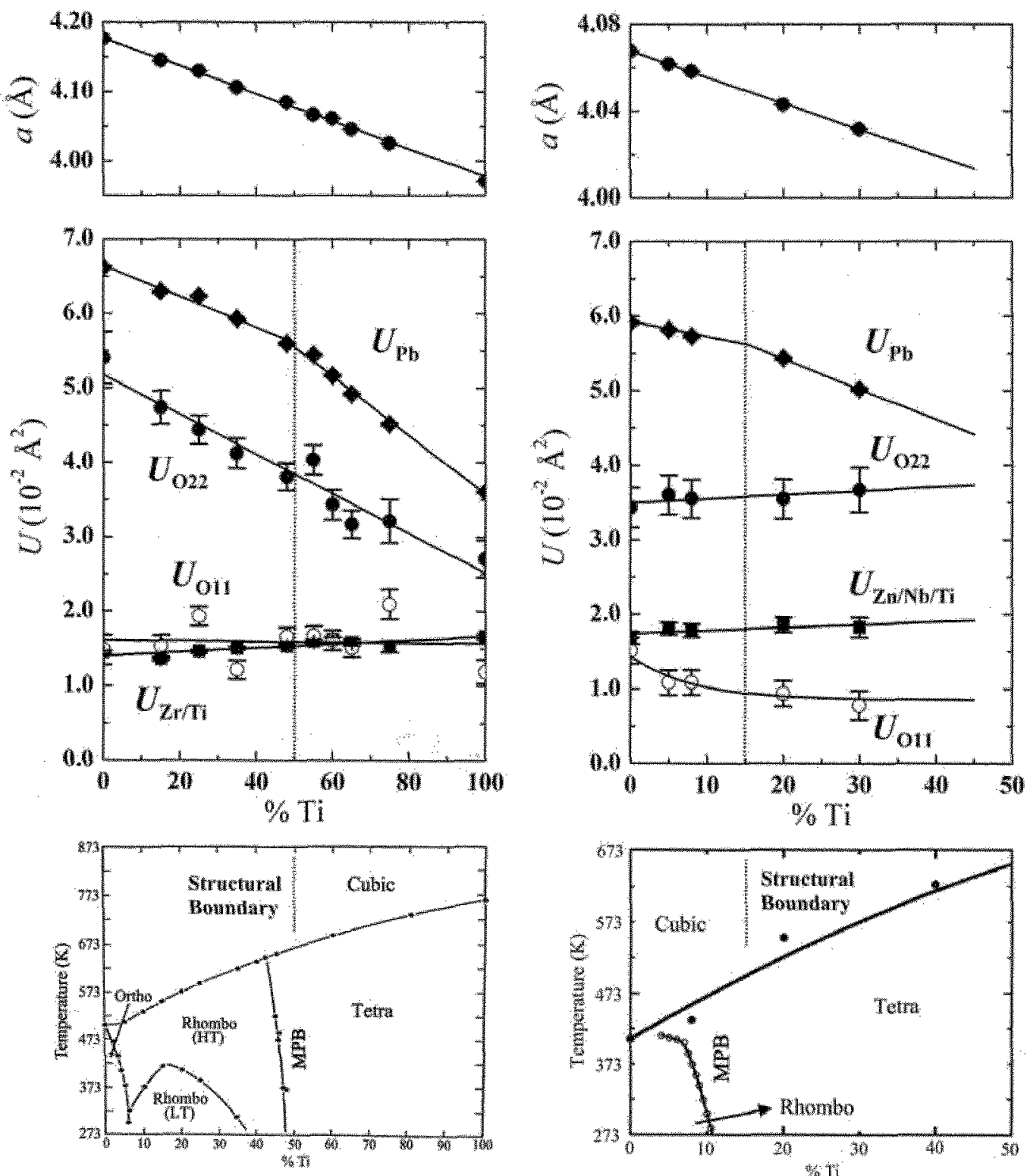

(a) PZT

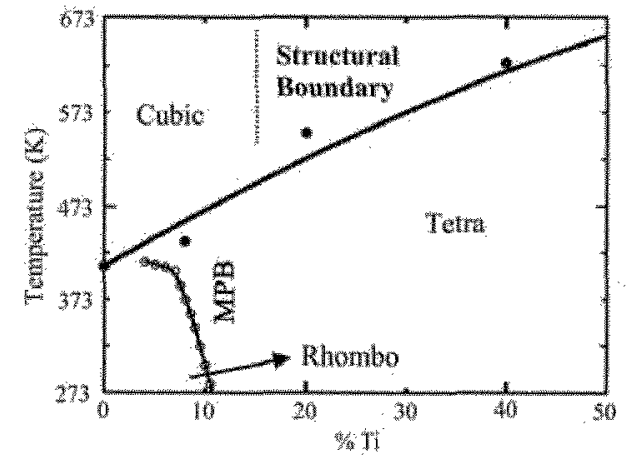

(b) PZN-PT

Fig. 3. Crystal structure parameters in cubic phase obtained by Rietveld analysis based on $\mathrm{Pb}$-ordered model. (a) $\mathrm{PZT}$ at $850 \mathrm{~K}$ and (b) PZN-PT at $650 \mathrm{~K}$. Here, $a$ is lattice parameter, and $U_{\mathrm{Pb}}, U_{\mathrm{Z} \times T \mathrm{Ti}}$ and $U_{\mathrm{Zn} / \mathrm{Nb} / \mathrm{Ti}}$ are isotropic mean square thermal displacement parameters. $U_{0}$ is expanded to anisotropic parameters $U_{011}$ and $U_{022}$. $U_{011}$ is parameter related to the thermal motion along the $\mathrm{Zr} / \mathrm{Ti}-\mathrm{O}$ or $\mathrm{Zn} / \mathrm{Nb} / \mathrm{Ti}-\mathrm{O}$ bond, whereas $U_{\mathrm{O} 22}$ is the parameter perpendicular to the bond. $U_{\mathrm{Pb}}$ changes abruptly around $50 \% \mathrm{Ti}$ content for $\mathrm{PZT}$ and $15 \% \mathrm{Ti}$ content for $\mathrm{PZN}-\mathrm{PT}$, where the disordered directions of the $\mathrm{Pb}$ atom are also changed. The structural boundary detected in the cubic phase is indicated by a dotted line in the phase diagram [1,3]. It is as if the structural boundary lies just above MPB. 


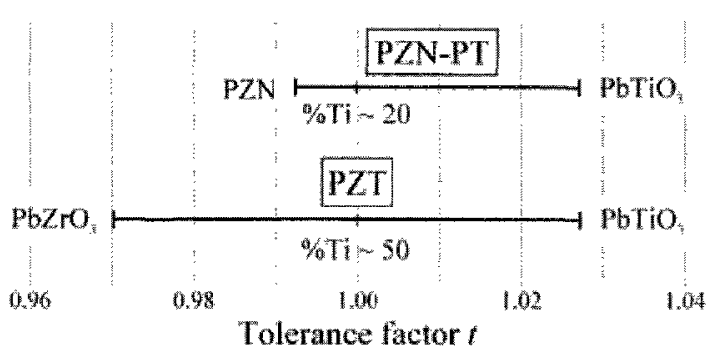

Fig. 4. Range of tolerance factor in PZT and PZN-PT systems. The ideal tolerance $t=1$ is achieved around $50 \% \mathrm{Ti}$ content and $20 \% \mathrm{Ti}$ content for $\mathrm{PZT}$ and PZN-PT, respectively. These Ti contents should give a close relation to the $\mathrm{Ti}$ content where the structural boundary and MPB.

is attributed to the change in thermal motions of the $\mathrm{Pb}$ atom, we consider that the local environment around the $\mathrm{Pb}$ atom must be changed dramatically at the composition. The tolerance factor is one of the indicators for the local atomic environments of perovskite-type oxides with the chemical formula of $A B \mathrm{O}_{3}$. It is defined as $t=\left(r_{A}+r_{0}\right) / \sqrt{2}\left(r_{B}+r_{0}\right)$, where $r_{A}$, $r_{B}$ and $r_{O}$ are the ionic radii of the $A$-site, $B$-site and $O$ atoms, respectively. The value $t<1$ means that the $A$-site environments are ample compared with $B$-site environments, whereas $t>1$ gives the opposite meaning. In Fig. 4, we show the range of tolerance factor in the PZT and PZN-PT systems. The ideal tolerance $t=1$, calculated by Shannon's ionic radii [11], is achieved around $50 \%$ Ti content for PZT and 20\% Ti content for PZN-PT. These compositions exactly correspond to the structural boundaries detected in the cubic phase. We suggest that in the $\mathrm{Pb}$-based perovskite-type solid solutions, the $B$-site atom substitution influences the structural fluctuation of the $\mathrm{Pb}$ atom, unlike of the $B$-site atom. The formation of MPB is strongly related to the change in the local environment around the $\mathrm{Pb}$ atom.

\section{CONCLUSIONS}

High-energy SR powder diffraction experiments give evidence that the thermal motion of the $\mathrm{Pb}$ atom in the cubic phase of PZT and PZN-PT solid solutions is changed at $50 \%$ and $15 \%$ Ti content, respectively. The change in the local environment around the $\mathrm{Pb}$ atom is considered to be a clue to the existence of MPB in $\mathrm{Pb}$-based perovskite-type solid solutions. Further systematic studies of cubic structures in a broad variety of perovskite-type solid solutions will provide information essential for a better understanding of phase transitions and for designing promising materials for applications based on accurate crystal structure physics.

\section{ACKNOWLEDGMENTS}

The authors thank Dr. E. Nishibori, Dr. M. Takata and Professor M. Sakata for their help in the MEM/Rietveld analysis. The SR experiment at SPring- 8 was carried out with the approval of the Japan Synchrotron Radiation Research Institute (JASRI) (Proposal No. 2005B7002 and 2007B0096).

\section{REFERENCES}

[1] B. Jaffe, W. R. Cook Jr. and H. Jaffe, "Piezoelectric Ceramics", Academic Press, London (1971) pp.136.

[2] M. Iwata, H. Hoshino, H. Orihara, Y. Ishibashi, H. Ohwa and N. Yasuda, Jpn. J. Appl. Phys., 39, 5156 (2000).

[3] M. Iwata, N. Tomisato, H. Orihara, N. Arai, N. Tanaka, H. Ohwa, N. Yasuda and Y. Ishibashi, Jpn. J. Appl. Phys., 40, 5819 (2001).

[4] S. Aoyagi, Y. Kuroiwa, A. Sawada, H. Tanaka, J. Harada, E. Nishibori, M. Takata and M. Sakata, J. Phys. Soc. Jpn., 71, 2353 (2002).

[5] I. Grinberg, V. R. Cooper and A. M. Rappe, Nature, 419, 909 (2002).

[6] Y. Kuroiwa, Y. Terado, S. J. Kim, A. Sawada, Y. Yamamura, S. Aoyagi, E. Nishibori, M. Sakata and M. Takata, Jpn. J. Appl. Phys., 44, 7151 (2005).

[7] Y. Terado, S. J. Kim, C. Moriyoshi, Y. Kuroiwa, M. Iawata, and M. Takata, Jpn. J. Appl. Phys., 45, 7552 (2006).

[8] M. Iwata, K. Katsuraya, S. Tachizaki, J. Hlinka, I. Suzuki, M. Maeda, N. Yasuda and Y. Ishibashi, Jpn. J. Appl. Phys., 43, 6812 (2004).

[9] E. Nishibori, M. Takata, K. Kato, M. Sakata, Y. Kubota, S. Aoyagi, Y. Kuroiwa, M. Yamakata and N. Ikeda, Nucl. Instrum. \& Methods Phys. Res., Sect. A, 467-468, 1045 (2001).

[10] M. Takata, E. Nishibori and M. Sakata, Z. Kristallogr., 216, 71 (2001).

[11] R. D. Shannon, Acta Crystallogr. A, 32, 751 (1976).

(Received December 7, 2007; Accepted December 17, 2007) 